\title{
Efficient Design of the Microstrip Reflectarray Antenna by Optimizing the Reflection Phase Curve
}

\author{
Xing Chen, Qiang Chen, Pan Feng, and Kama Huang \\ College of Electronic and Information Engineering, Sichuan University, Chengdu 610064, China \\ Correspondence should be addressed to Xing Chen; xingc@live.cn
}

Received 5 January 2016; Accepted 27 March 2016

Academic Editor: Giuseppe Mazzarella

Copyright ( 2016 Xing Chen et al. This is an open access article distributed under the Creative Commons Attribution License, which permits unrestricted use, distribution, and reproduction in any medium, provided the original work is properly cited.

\begin{abstract}
The microstrip reflectarray antenna is an attractive directional antenna combining advantages of both the reflector and the microstrip array antenna. For the design of this kind of antenna, the conventional method, which is based on the reflection phase curve, may not be effective because of the approximations made in the design procedure. The common optimization method that employs an optimization algorithm in conjunction with the full-wave simulation to optimize the antennas' structural parameters is able to achieve better performances in comparison with the conventional method; but it is impractical for large-scale microstrip reflectarray antennas due to too many structural parameters from variable reflection elements. To tackle the design problem of the microstrip reflectarray antenna, a new method is proposed for the first time. It optimizes the reflection phase curve rather than the structural parameters and then utilizes the optimized reflection phase curve to design the antenna. A microstrip reflectarray antenna working at $5.8 \mathrm{GHz}$ and with a fixed size of $300 \mathrm{~mm} \times 300 \mathrm{~mm}$ is designed for high gain as a sample design. The results show that the proposed method has greatly improved the antenna's gain over $2.2 \mathrm{~dB}$ (from $19.7 \mathrm{dBi}$ to $21.9 \mathrm{dBi}$ ) in comparison with the conventional design method, and it only needs to optimize 6 structural parameters, in contrast to 22 for the common optimization method.
\end{abstract}

\section{Introduction}

The microstrip reflectarray antenna proposed by Malagisi [1] has raised significant interest in the high-gain antenna field, due to its salient features combining some of the best features of reflector and array antennas [2]. It provides an attractive alternative to conventional directive antennas and has been widely applied in many fields recently, for example, direct broadcast satellite (DBS), space telemetry, and radar applications [3-5].

A microstrip reflectarray antenna usually consists of a feed source and a reflector. The feed source is an antenna placed at a certain distance from the reflector, and the reflector is an array of microstrip patches and/or slots etched on a grounded dielectric substrate. Microwave energy is emitted from the feed source and scattered by the reflector. Elements in the reflector are designed to generate proper phase compensations associated with the path lengths from the feed source so that a planar phase surface is formed in front of the aperture of the antenna, and in this way the directional radiation property with high gain can be achieved.

The conventional design method for the microstrip reflectarray antenna contains the following major steps: analyzing the phase characteristics of the reflection elements using numerical simulations to obtain a reflection phase curve, which describes the one-to-one correspondence between the phase compensation and a structural parameter that controls the elements' reflection phase; calculating the required phase compensation for each reflection element according to the distance and the relative location between the reflection element and the feeding source; and finally determining the structural parameters of the reflection elements based on the reflection phase curve and the required phase compensation $[2,6-8]$.

This conventional design method is simple and has been adopted for most microstrip reflectarray antennas. However, its design may not be an optimum one because of the approximations made in the design procedure as described 
as follows. The reflection phase curve is obtained under the assumption of a normal incidence [9], but actually the incident angle may vary and the reflection phase curves are different with different incident angles [10]. The feed source and the reflection elements are considered as sizeless points in the calculation of the path length for determining the phase compensation [2]. The coupling effect between the reflection elements is ignored in the design procedure but it actually may not be negligible when the distance between patch edges is small (e.g., less than a quarter wavelength) $[2,11]$. The field diffracted by the reflector's edges, the shielding effect of the feed source, and the support frame are not taken into account in the design procedure $[2,12]$. Those approximations may bring forth errors in the design and subsequently deteriorate the performances of the antennas designed.

A widely used method for antenna design employs an optimization algorithm such as the genetic algorithm (GA) in conjunction with the full-wave simulation to optimize the antenna's structural parameters [13-16]. The optimization algorithm enables design of antennas with optimum performance, and the full-wave simulation allows taking all the involved effects into account without the abovementioned approximations. This method was employed in the authors' previous work [17] to design a microstrip reflectarray antenna consisting of $7 \times 7$ reflection elements, and encouraging results were achieved; for example, the antenna's gain has been considerably improved over $1.4 \mathrm{dBi}$ (from $18.1 \mathrm{dBi}$ to $19.5 \mathrm{dBi}$ ) in comparison with the conventional design method.

However, the aforementioned common optimization method is impractical for a large-scale microstrip reflectarray because its large number of structural parameters deriving from its variable reflection elements would result in too many unknown parameters needed in the optimization. Taking the microstrip reflectarray studied in [17] as an example, the number of the unknown structural parameters is 10 for the antenna containing $7 \times 7$ reflection elements, but it will become 27 if the quantity of the reflection elements rises to $13 \times 13$. In practical applications, microstrip reflectarray antennas are usually of much more reflection elements, which would result in not only a huge computation burden but also a difficulty of achieving good results using an optimization algorithm. That is why very little work published in literature has employed the optimization method to design a microstrip reflectarray antenna.

To solve the design problem of microstrip reflectarray antennas, this paper proposes a novel method, which employs an optimization algorithm in conjunction with the full-wave simulation to optimize the reflection phase curve rather than the structural parameters and then uses the optimized reflection phase curve to design a microstrip reflectarray antenna. This method is able to make full use of the advantages of the optimization algorithm and the full-wave simulation so as to achieve better design in comparison with the conventional method. Meanwhile, it can reduce the number of unknown parameters needed in the optimization to a low level and thus possesses the capacity of effectively designing large-scale microstrip reflectarray antennas.
The other sections of this paper are organized as the follows. Section 2 introduces the configuration of the microstrip reflectarray antenna to be designed in this work. The conventional design method is described and its design results are given in Section 3. The principle and procedure of the proposed design method are presented in Section 4. Finally, in Section 5, conclusions are drawn.

\section{Antenna Configuration}

To validate the proposed design method, it is employed to design a sample $300 \mathrm{~mm} \times 300 \mathrm{~mm}$ microstrip reflectarray antenna operating at $5.8 \mathrm{GHz}$ with high gain and end-fire property (i.e., the main beam of the antenna is set to be perpendicular to the reflector). As illustrated in Figure 1(a), the feed source of the antenna is a rectangular patch antenna, whose configuration and parameters are shown in Figure 1(b). It is propped by three metal sticks and placed at a distance of $h_{1}$ from the reflector. The reflector consists of a metal ground fabricated by an aluminum board with thickness of $4.0 \mathrm{~mm}$ and a printed circuit board (PCB) with relative dielectric constant $\varepsilon_{r}=2.65$ and thickness $h_{2}=$ $1 \mathrm{~mm}$. The PCB and the metal ground are separated by an air layer with thickness $h_{3}$.

As illustrated in Figure 1(c), various reflection elements are etched on the PCB. Because of the geometrical symmetry and the antenna's end-fire property, the reflection elements whose distances to the center of the reflector are equal should be of the same reflection phase and thus the same structural parameters. Hence, the reflection elements can be indexed from 1 to $n$ according to their distance to the center where $n$ is the quantity of groups of the elements with different dimensions, as illustrated in Figure 1(c).

As shown in Figure 1(d), each element contains a square patch with a side length $L_{1}$ as well as a square ring with an outer side length $L_{2}$ and thickness $W$, and it occupies a square area $L * L$. To simplify the structure of the reflection element, in this work, $L_{1}$ and $W$ are taken as $L_{1}(i)=k_{1} * L_{2}(i)$ and $W(i)=k_{2} * L_{2}(i)$, where $k_{1}$ and $k_{2}$ are two constant coefficients and $i$ is the index number of the element. Thus, for a reflection element, among its structural parameters $L_{1}, L_{2}$, and $W$, only one of them is independent from the others. In this work, $L_{2}$ is selected to represent the element dimension.

\section{Design Using the Conventional Design Method}

To provide a benchmark for comparison with and an initial design for the proposed method, the conventional design method is employed to design the microstrip reflectarray antenna introduced in the last section.

The side length $L$ of the square area occupied by a reflection element, which is also the distance between the adjacent element centers, is set to be $0.6 \lambda$, where $\lambda$ is the free-space wavelength at the working frequency $5.8 \mathrm{GHz}$. This setting can help to avoid the appearance of grating lobes to a certain extent [2]. For a reflectarray antenna, the distance $h_{1}$ is required to be large enough so that the incident wave to 


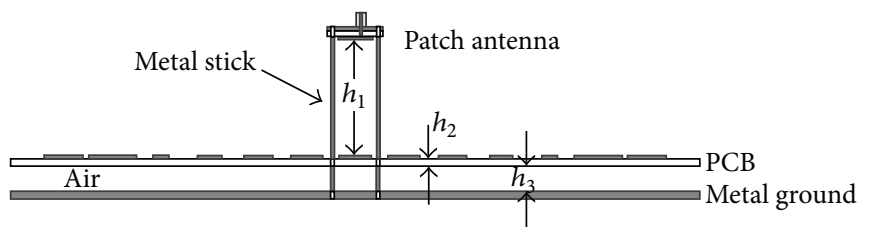

Metal

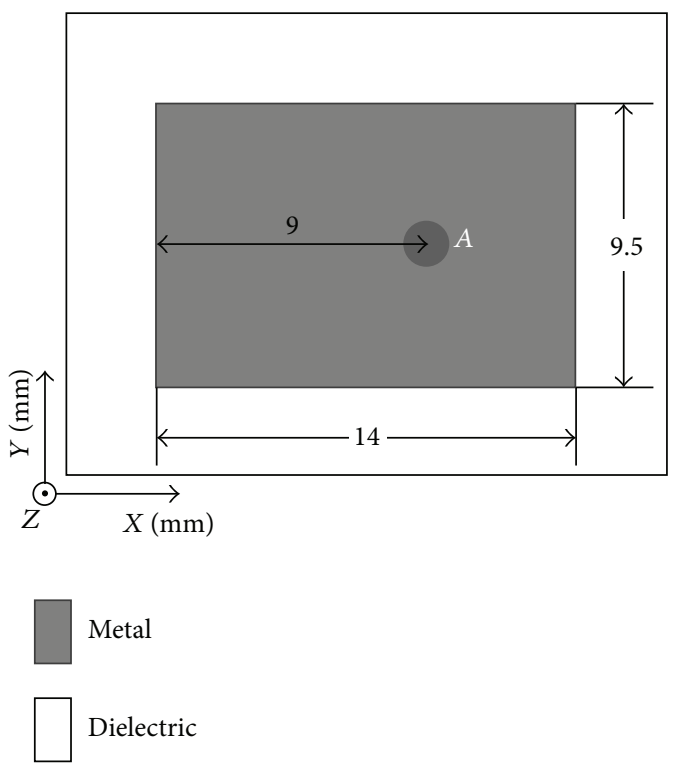

(b) (a)

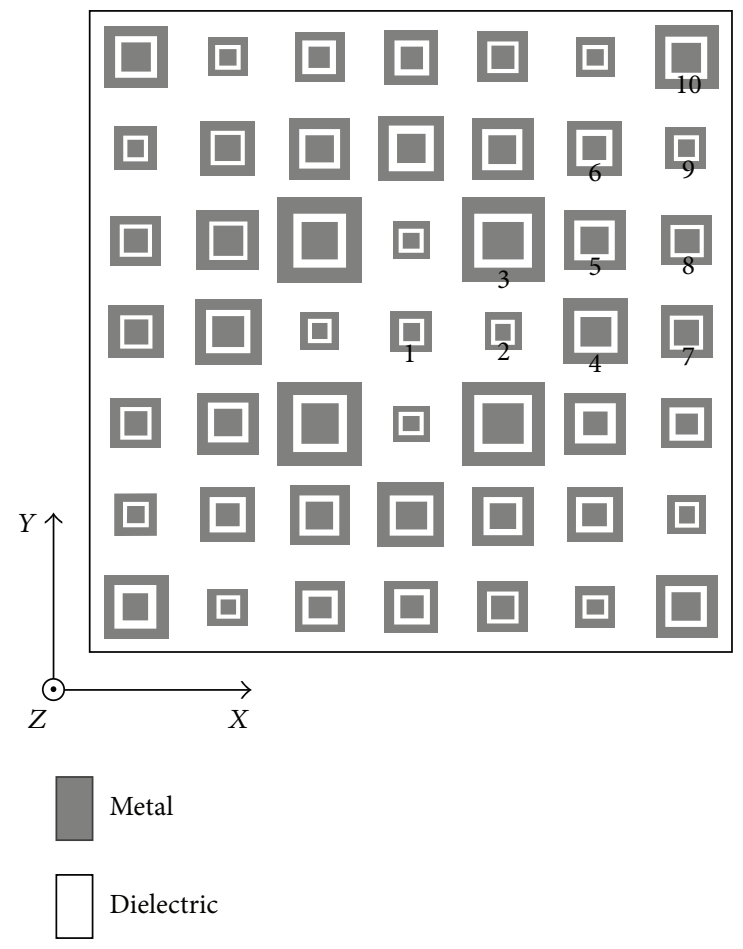

(c)

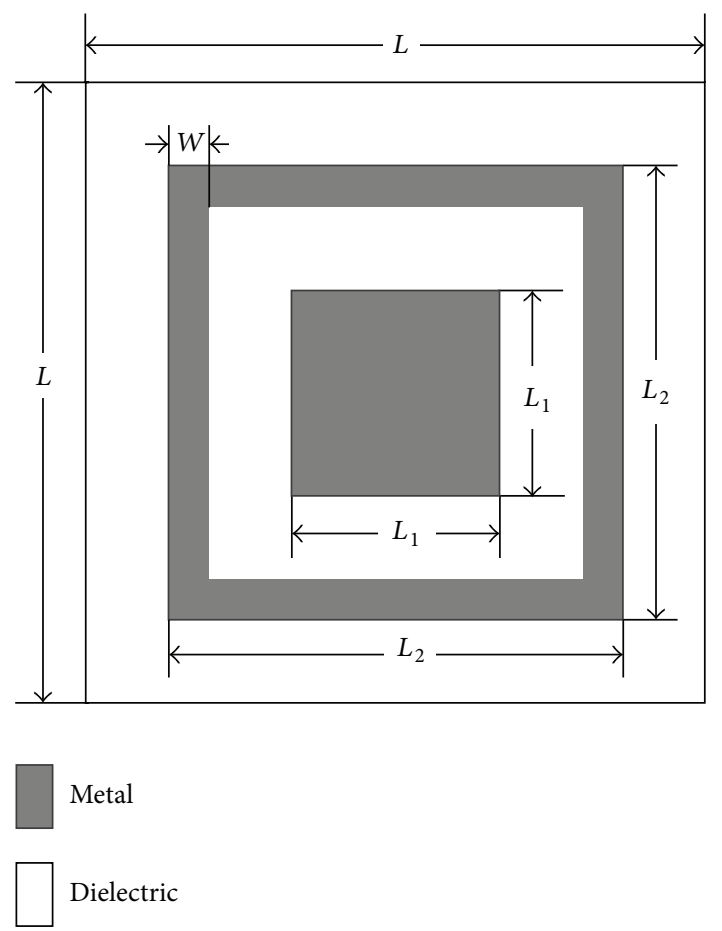

(d)

FIGURE 1: The configuration of the microstrip reflectarray antenna: (a) side view of the reflectarray antenna; (b) top view of the patch antenna; (c) top view of the reflector; (d) configuration of a reflection element.

the reflection elements can be approximated as a plane wave $[10,18]$, but $h_{1}$ cannot be too large in order to ensure that the reflector covers at least the main lobe of the feed source. As a compromise, $h_{1}$ is set to be $100 \mathrm{~mm}$ in this work.

The reflection phase curve plays a key role in the conventional method for designing a microstrip reflectarray antenna. To compensate various phases, the reflection phase curve should cover a phase range of 360 degrees, and meanwhile the curve should be monotonically smooth in order to reduce the fabrication sensitivity [2]. After a serial of parameter sweeps using the full-wave simulation and employing the method presented in [9] for analyzing the reflection characteristics of the reflection elements, a monotonically smooth reflection phase curve is derived and presented in Figure 2 for $k_{1}=0.5, k_{2}=0.15$, and $h_{3}=12 \mathrm{~mm}$. In this work, the full-wave simulation is 


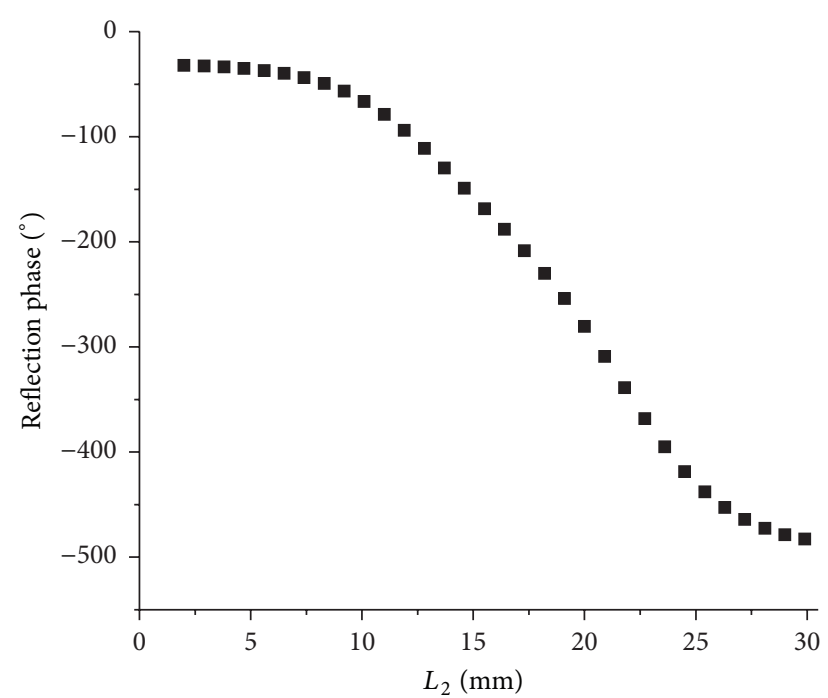

FIgURE 2: The reflection phase versus the square ring outer length $L_{2}$.

conducted by employing the popular commercial software CST MICROWAVE STUDIO (MWS) based on the finite integration technique (FIT).

Then, the required phase compensation for each reflection element is calculated using the equation [13]

$$
\phi_{i}=k\left(R_{i}-\overrightarrow{\mathbf{r}_{\mathbf{i}}} \cdot \overrightarrow{\mathbf{r}_{\mathbf{0}}}\right) \text {, }
$$

where $k$ is the propagation constant in vacuum, which is equal to $2 \pi / \lambda ; R_{i}$ is the distance from the phase center of the feed source to $i$ th element; $\overrightarrow{\mathbf{r}_{\mathbf{i}}}$ is the vector from the center of the reflectarray to the element; and $\overrightarrow{\mathbf{r}_{\mathbf{0}}}$ is the unit vector in the main beam direction.

In this work, the main beam of the antenna is set to be perpendicular to the reflector so that the angle between $\overrightarrow{\mathbf{r}_{\mathbf{i}}}$ and $\overrightarrow{\mathbf{r}_{\mathbf{0}}}$ is 90 degrees making the dot product $\overrightarrow{\mathbf{r}_{\mathbf{i}}} \cdot \overrightarrow{\mathbf{r}_{\mathbf{0}}}$ zero. Then, (1) is simplified to

$$
\phi_{i}=k R_{i}
$$

Because the size of the reflector is fixed to be $300 \mathrm{~mm} \times$ $300 \mathrm{~mm}$ and the element spacing $L$ is $0.6 \lambda \approx 31 \mathrm{~mm}$, the reflector can only contain a maximum of $9 \times 9$ reflection elements. The reflection elements have 15 different sizes. According to (2), the required reflection phase for those elements are (in degree) $-24,-351.3,-319.9,-260.8,-232.8$, $-153.9,-129,-104.8,-35.5,-289.3,-330.5,-309.7,-249.4$, -155.2 , and -33.8 , respectively. By referring to the reflection phase curve in Figure 2, the square ring outer length $L_{2}(i)$ can be determined as follows (in mm): $L_{2}(1)=23.22, L_{2}(2)=$ 22.17, $L_{2}(3)=21.24, L_{2}(4)=19.35, L_{2}(5)=18.3, L_{2}(6)=$ $14.82, L_{2}(7)=13.68, L_{2}(8)=12.48, L_{2}(9)=23.61, L_{2}(10)=$ 20.28, $L_{2}(11)=21.54, L_{2}(12)=20.91, L_{2}(13)=18.93$, $L_{2}(14)=14.88$, and $L_{2}(15)=23.55$.

For this microstrip reflectarray designed using the conventional method, its characteristics are simulated by CST and shown in Figures 3 and 4. One observes from Figure 3

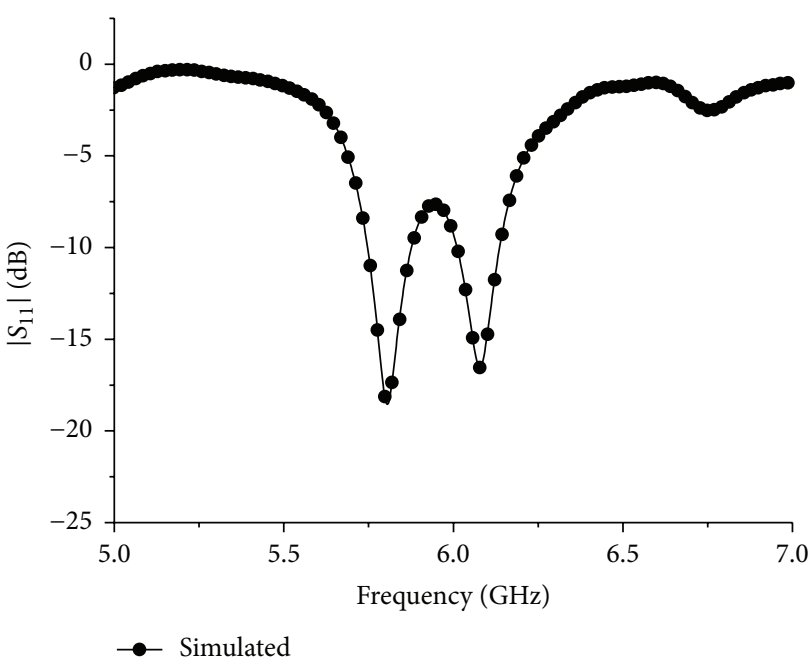

FIGURE 3: Simulated $\left|S_{11}\right|$ for the microstrip reflectarray designed by the conventional method.

that the simulated $\left|S_{11}\right|$ is $-18.4 \mathrm{~dB}$ at the working frequency of $5.8 \mathrm{GHz}$ and $\left|S_{11}\right|<-10 \mathrm{~dB}$ impedance bandwidth is about 2.2\% (from $5.75 \mathrm{GHz}$ to $5.88 \mathrm{GHz}$ ) and Figure 4 illustrates that the antenna has good end-fire property at $5.8 \mathrm{GHz}$, its gain is $19.7 \mathrm{dBi}$, and side-lobes are about $12.3 \mathrm{~dB}$ below the main lobe.

It is worth noting from the simulation results that the three metal sticks have very strong effect on the antenna's radiation properties. But the conventional design method cannot take into consideration the shielding effect of the metal sticks and just simply ignored it. According to the CST simulation, the gain of this antenna should go up to $21.5 \mathrm{dBi}$, $1.8 \mathrm{~dB}$ over the gain of $19.7 \mathrm{~dB}$, if the three metal sticks are taken away. This is a good example demonstrating the defect of the conventional design method.

\section{The Proposed Design Method by Optimizing the Reflection Phase Curve}

4.1. Principle of the Proposed Design Method. As discussed above, for the microstrip reflectarray antenna, the conventional design method may not be effective due to the approximations made in the design procedure. But the common optimization method, which employs an optimization algorithm in conjunction with the full-wave simulation to optimize antennas' structural parameters, is impractical for largescale microstrip reflectarray antennas because of too many unknown parameters from various elements in the reflector. In this section, a new design method, which is effective and with acceptable computation burden, is proposed for the first time for the microstrip reflectarray antenna.

The reflection phase curve plays a key role in the microstrip reflectarray antenna design. Using a reflection phase curve, the structural parameters of the reflection elements can be determined according to the required phase compensation of those elements. Hence, one would expect that optimizing a reflection phase curve may result in the same effect on the antenna designed as optimizing 


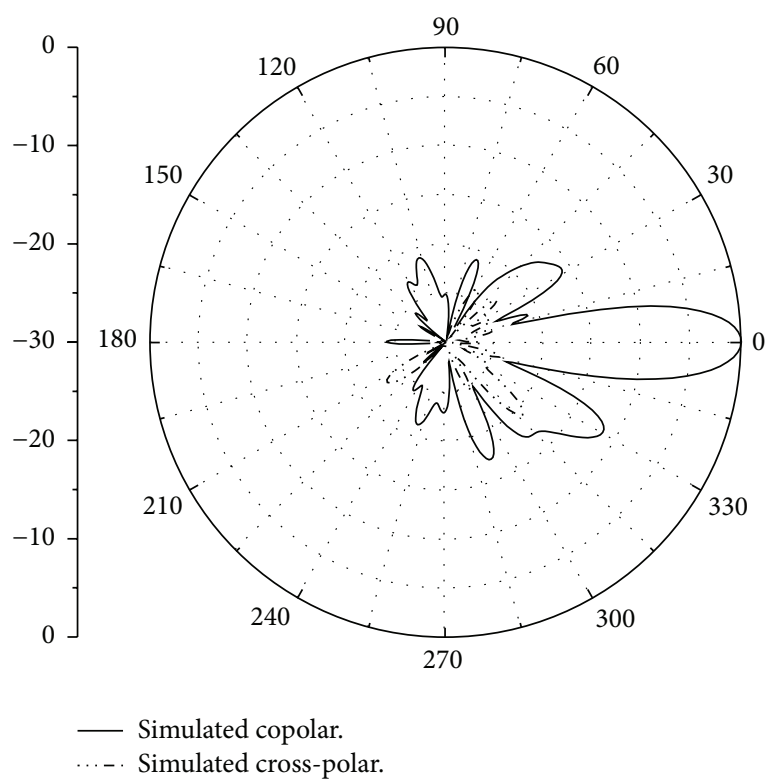

(a)

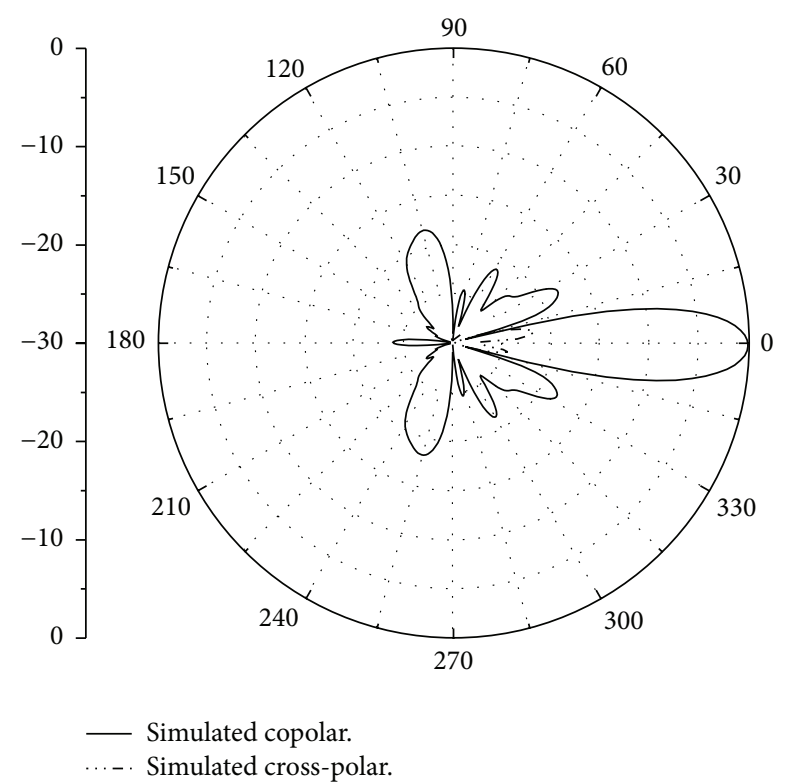

(b)

FIGURE 4: Simulated radiation patterns on the $X Z$ plane and $Y Z$ plane: (a) $X Z$ plane; (b) $Y Z$ plane.

the structural parameters. Moreover, the reflection phase curve is a continuous curve. Its continuity allows using only a few parameters to describe it, and thus the unknown parameters needed in the optimization can be controlled to a very low level even for a large-scale microstrip reflectarray antenna.

To validate the proposed design method, the microstrip reflectarray antenna introduced in Section II is designed as an example. In the design procedure, an optimization algorithm, that is, the genetic algorithm (GA), in conjunction with the full-wave simulation is employed for optimizing the reflection phase curve; and then the optimized curve is used to design the microstrip reflectarray antenna for high performances.

4.2. Design Procedure. The GA is a powerful and efficient optimization technique, which makes use of a natural evolution process: the improvement of a population of parameters along with successive generations by applying the genetic operators, for example, mutation, selection, and crossover. The GA is adopted in this paper to optimize the reflection phase curve of the microwave reflectarray antenna.

A reflection phase curve is typically "S" shaped. In this work we use the DoseResp Function defined in the following to fit the reflection phase curve:

$$
f(x)=A_{1}+\frac{A_{2}-A_{1}}{1+10^{(\operatorname{Mid}-x) * p}},
$$

where $f$ is the phase compensation value, $x$ represents the structural parameters that control the elements' reflection phase, and $A_{1}, A_{2}$, Mid, and $p$ are 4 unknown parameters.

By using a curve fitting tool available in Matlab, the reflection phase curve shown in Figure 2 can be expressed by the DoseResp Function given in (3) for $A_{1}=-536.34$, $A_{2}=-19.61, \mathrm{Mid}=19.63$, and $p=-0.101$.

Besides $A_{1}, A_{2}$, Mid, and $p$, the parameters $L$ and $h_{1}$ are also taken into the optimization for better results. Then, there are 6 unknown parameters that need to be optimized. For those unknown parameters, the determination of their possible value range is very important to the GA optimization because it has a big impact on the optimization efficiency and results. These parameters' initial values can be obtained from the design using the conventional method; and then after giving a considerable margin for the GA-based optimization, the parameters $L, h_{1}, A_{1}, A_{2}$, Mid, and $p$ are confined to be $0.35 \lambda \sim 0.85 \lambda, 70 \mathrm{~mm} \sim 130 \mathrm{~mm},-650 \sim-400,-100 \sim 100,0.02$ $\sim 30,-0.3 \sim-0.001$, respectively.

The goal of the GA optimization is to achieve a high gain and good impedance match at the working frequency of $5.8 \mathrm{GHz}$. Hence, the fitness function, which represents the desired performance requirements and guides the direction of the GA optimization, is defined as

$$
\text { Fitness }=C_{1} \times \text { MaxGain }+C_{2} \times \operatorname{Max} S 11 \text {, }
$$

where Fitness represents the value of the fitness function; MaxGain refers to the radiation gain at the working frequency of $5.8 \mathrm{GHz}$; and MaxS11 denotes the maximum $\left|S_{11}\right|$ over a preset frequency band ranging from 5.7 to $5.9 \mathrm{GHz}$. The values of the gain and $\left|S_{11}\right|$ are in $\mathrm{dB} . C_{1}$ and $C_{2}$ are weight coefficients, whose values should emphasize the relative importance of each term in the design requirements, but no specific rule exists for determining their values. In this work they are selected by experience and are set to be 0.03 and -0.02 , respectively.

A GA-based optimization is executed. In the optimization, the GA employs tournament selection with elitism, single-point crossover with probability $P_{c}=0.5$, and jump 


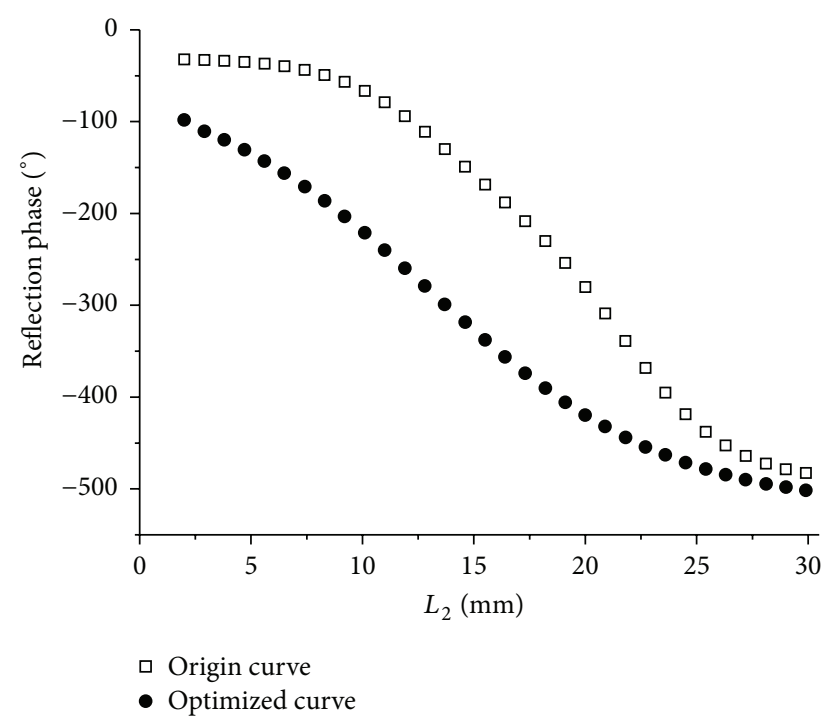

FIGURE 5: A comparison of the origin and the optimized reflection phase curve.

mutation with probability $P_{m}=0.2$, and it uses 50 generations and 100 individuals in a population.

In the design procedure, the performance of the antenna is simulated by full-wave simulations employing CST MWS. To reduce the heavy computational burden, the design procedure is parallelized in a master-slave model and implemented on a Beowulf cluster system [13]. The Beowulf cluster system consists of 32 processors interconnected by a fast $1000 \mathrm{Mb} / \mathrm{s}$ Ethernet. One processor, named the master processor, carries out the GA optimization while all the others, called slave processors, execute the full-wave simulations.

4.3. Results and Analysis. The parameters determined by the GA-based optimization are as follows: $L=0.409 \lambda, h_{1}=$ $95.75 \mathrm{~mm}, A_{1}=-521.34, A_{2}=-48$, Mid $=13.05$, and $p=-0.08$.

Because the optimized element spacing $L$ is $0.409 \lambda$, which is less than that in the conventional design described above, the reflector with fixed size of $300 \times 300 \mathrm{~mm}^{2}$ is able to contain $11 \times 11$ reflection elements. It can be seen that there are 20 reflection elements with different dimensions in the reflector of this antenna. Noticing that each different element has one unknown parameter, and adding the parameters co and $h_{1}$, there would be 22 unknown parameters in need for optimization if we utilize the common optimization method. Obviously, it is very difficult for an optimization algorithm, even for a powerful algorithm such as the GA, to achieve good results with so many parameters that need to be optimized.

In Figure 5, the optimized reflection phase curve is compared with the origin one. Based on the optimized reflection phase curve, the structural parameters of the reflection elements are determined; and then a prototype antenna is fabricated and depicted in Figure 6. To illustrate the dimension of the antenna, a ruler is placed in front of it in the figure.

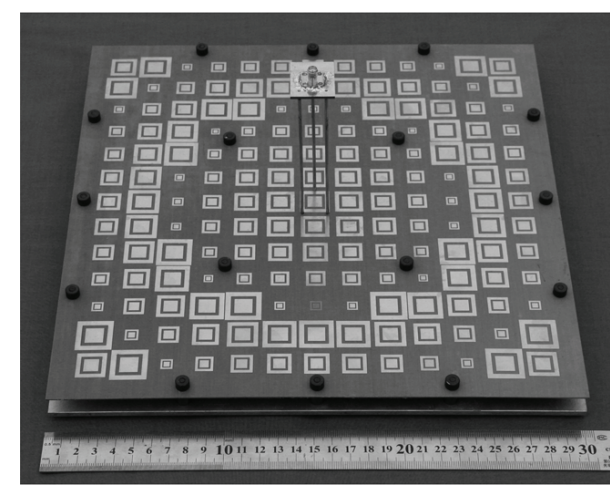

FIgURE 6: Prototype of the antenna.

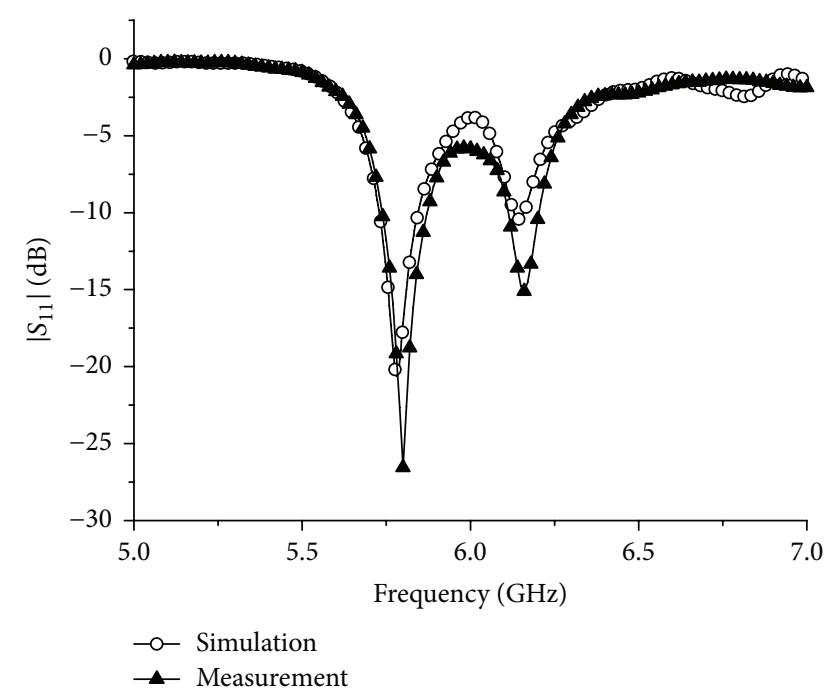

FIgURE 7: Measured and simulated $\left|S_{11}\right|$.

The reflection coefficient of the prototype antenna is measured with an Agilent E8362B vector network analyzer. In Figure 7, the measured and simulated $\left|S_{11}\right|$ curves are compared with each other and a good agreement is observed. Also, the figure illustrates that $\left|S_{11}\right|<-10 \mathrm{~dB}$ impedance bandwidth is about $2 \%$ (from $5.77 \mathrm{GHz}$ to $5.88 \mathrm{GHz}$ ), which is very close to that achieved by the conventional design method.

The radiation patterns of the proposed antenna are measured in an anechoic chamber, and the measurement results are compared with the simulated radiation patterns in the $X Z$ plane and $Y Z$ plane at $5.8 \mathrm{GHz}$. The comparison is shown in Figure 8, where one observes that they also agree very well and this antenna possesses good end-fire property. The measured side-lobes are about $18 \mathrm{~dB}$ below the main lobe, and the measured cross-polarization levels are less than $20 \mathrm{~dB}$ in both $X Z$ and $Y Z$ planes.

Figure 9 compares measured gains against frequencies of two antennas; one of them is designed by the conventional method and the other is designed by the proposed optimization method. The comparison shows that, in comparison with the conventional method, the proposed optimization 


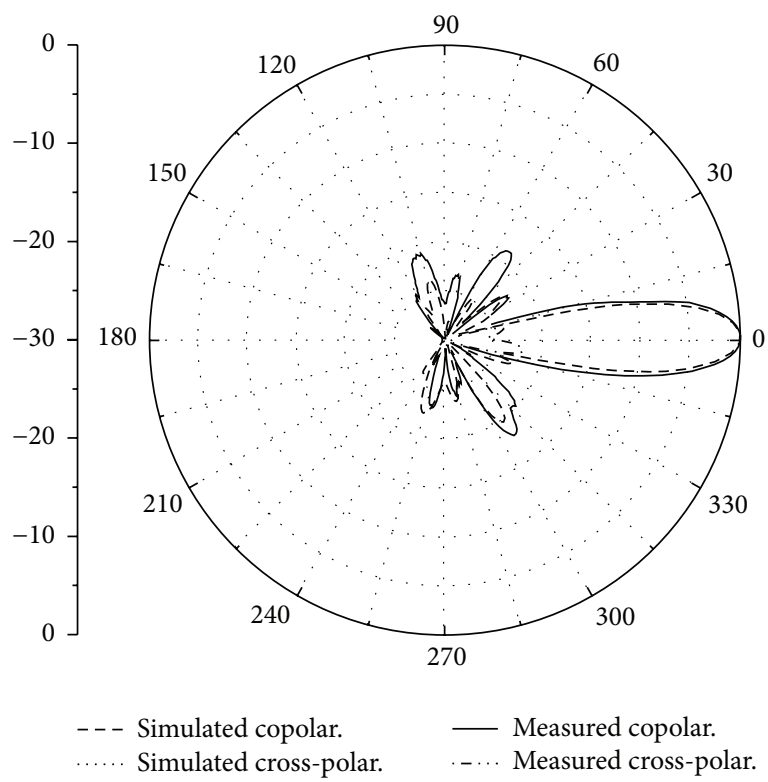

(a)

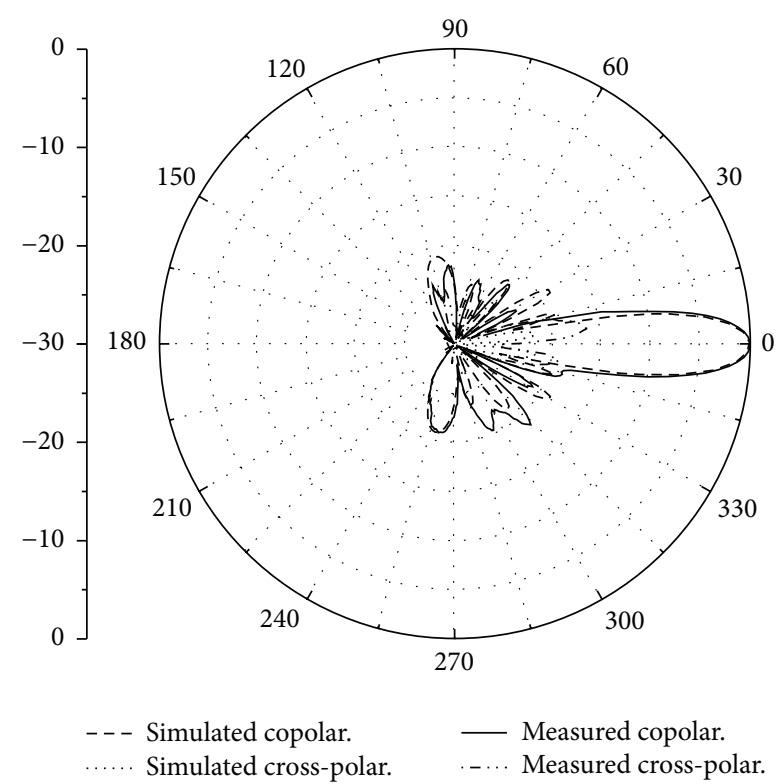

(b)

FIGURE 8: Measured and simulated radiation patterns on the $X Z$ plane and $Y Z$ plane: (a) $X Z$ plane; (b) $Y Z$ plane.

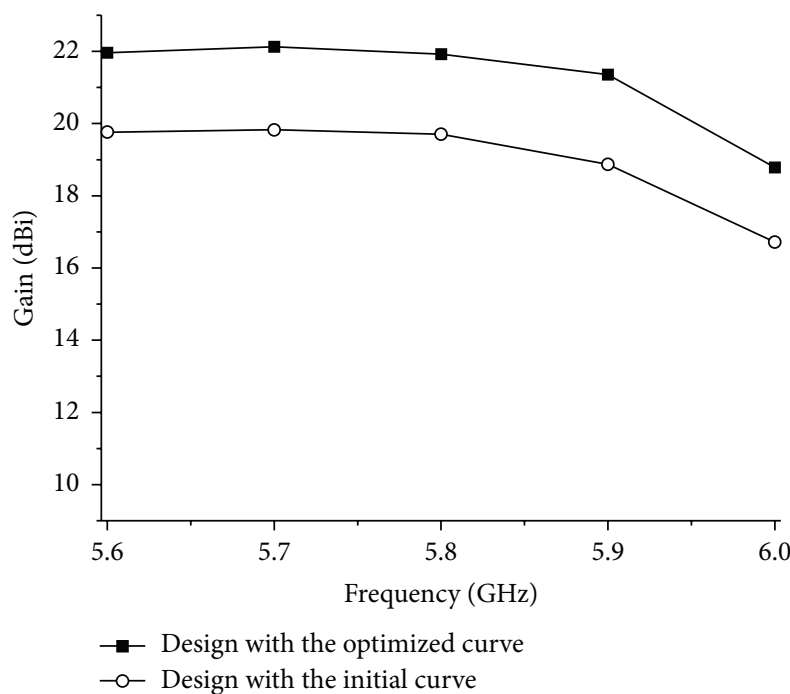

Figure 9: Gain comparison with frequency varying from 5.6 to $6.0 \mathrm{GHz}$

method has considerably improved the gain of the microstrip reflectarray antenna over the frequency band from $5.6 \mathrm{GHz}$ to $6.0 \mathrm{GHz}$; at the working frequency of $5.8 \mathrm{GHz}$, it achieves a gain of $21.9 \mathrm{~dB}$, which is $2.2 \mathrm{~dB}$ over that of the conventional design $(19.7 \mathrm{dBi})$ and even more than that of the conventional design without three metal sticks $(21.5 \mathrm{dBi})$. This demonstrates that the proposed optimization method is able to remedy or offset the adverse effects caused by some factors such as the support frame's shield.

\section{Conclusions}

Currently, the design of the microstrip reflectarray is under an embarrassing condition. The conventional design, which is adopted for most microstrip reflectarray antennas, may not be effective due to many approximations made in its design procedure, while the common optimization method that optimizes antennas' structural parameters is impractical for large-scale antenna because of too many parameters in need for optimization. A novel design method is proposed in this paper. It employs an optimization algorithm such as the GA in conjunction with the full-wave simulation to optimize the reflection phase curve and utilizes the optimized curve to design a microstrip reflectarray antenna.

As an example, the design of a microstrip reflectarray antenna with fixed size of $300 \times 300 \mathrm{~mm}^{2}$ for high gain is presented. The results demonstrate that the proposed method is feasible and effective. Being able to avoid the approximations made in the conventional design, it can greatly improve the antenna's gain over $2.2 \mathrm{~dB}$ (from $19.7 \mathrm{dBi}$ to $21.9 \mathrm{dBi}$ ). By controlling the number of the unknown parameters in need for optimization to a low level, the proposed method only need to optimize 6 parameters, but the common optimization method has to optimize 22 parameters. Hence, the proposed method has the ability of designing large-scale antennas. These merits will make the proposed method to be a powerful tool for the design of microstrip reflectarray antennas.

\section{Competing Interests}

There is no conflict of interests related to this paper. 


\section{Acknowledgments}

This work was supported by the China NSAF Fund under Grant U1230112.

\section{References}

[1] C. S. Malagisi, "Microstrip disc element reflectarray," in Proceedings of the Electronics and Aerospace Systems Convention, vol. 1, pp. 186-192, Arlington, Va, USA, September 1978.

[2] J. Huang and J. A. Encinar, Reflectarray Antennas, Wiley Interscience, 2007.

[3] J. A. Encinar, M. Arrebola, M. Dejus, and C. Jouve, "Design of a 1-metre reflectarray for DBS application with 15\% bandwidth," in Proceedings of the 1st European Conference on Antennas and Propagation (EuCAP '06), pp. 1-5, Nice, France, November 2006.

[4] C. Cheymol, T. Dousset, P. Dumon, M. Labeyrie, and C. Renard, "A X-band electronically scanned reflectarray antenna for space telemetry," in Proceedings of the European Conference on Antennas and Propagation (EuCAP '03), pp. 2766-2668, Berlin, Germany, November 2009.

[5] J. Huang, "Microstrip reflectarray antenna for the SCANSCAT radar application," JPL Publication 90-45, 1990.

[6] H. Li, B.-Z. Wang, G. Zheng, W. Shao, and L. Guo, "A reflectarray antenna backed on FSS for low RCS and high radiation performances," Progress In Electromagnetics Research C, vol. 15, pp. 145-155, 2010.

[7] J. Ethier, M. R. Chaharmir, and J. Shaker, "Reflectarray design comprised of sub-wavelength coupled-resonant square loop elements," Electronics Letters, vol. 47, no. 22, pp. 1215-1217, 2011.

[8] S. Costanzo, "Reflectarray antennas: analysis and synthesis techniques," International Journal of Antennas and Propagation, vol. 2012, Article ID 945682, 3 pages, 2012.

[9] F.-C. E. Tsai and M. E. Bialkowski, "An equivalent waveguide approach to designing of reflect arrays with the use of variablesize microstrip patches," Microwave and Optical Technology Letters, vol. 34, no. 3, pp. 172-175, 2002.

[10] S. D. Targonski and D. M. Pozar, "Analysis and design of a microstrip reflectarray using patches of variable size," in Proceedings of the IEEE Antennas and Propagation International Symposium, pp. 1820-1823, IEEE, June 1994.

[11] R. D. Javor, X.-D. Wu, and K. Chang, "Design and performance of a microstrip reflectarray antenna," IEEE Transactions on Antennas and Propagation, vol. 43, no. 9, pp. 932-939, 1995.

[12] R. E. Zich, M. Mussetta, M. Tovaglieri, P. Pirinoli, and M. Orefice, "Genetic optimization of microstrip reflectarrays," in Proceedings of the Antennas and Propagation Society International Symposium, pp. 128-131, San Antonio, Tex, USA, June 2002.

[13] X. Chen, K. Huang, and X.-B. Xu, "Automated design of a threedimensional fishbone antenna using parallel genetic algorithm and NEC," IEEE Antennas and Wireless Propagation Letters, vol. 4, no. 1, pp. 425-428, 2005.

[14] J. M. Jayasinghe, J. Anguera, D. N. Uduwawala, and A. Andújar, "Nonuniform overlapping method in designing microstrip patch antennas using genetic algorithm optimization," International Journal of Antennas and Propagation, vol. 2015, Article ID 805820, 8 pages, 2015.

[15] K. Michael and A. Kucharski, "Genetic algorithm optimization for multiband patch antenna design," in Proceedings of the 1st
European Conference on Antennas and Propagation (EuCAP '06), pp. 1-4, Nice, France, November 2006.

[16] M. Rattan, M. S. Patterh, and B. S. Sohi, "Optimization of gain, impedance, and bandwidth of Yagi-Uda array using particle swarm optimization," International Journal of Antennas and Propagation, vol. 2008, Article ID 193242, 4 pages, 2008.

[17] Y. Chen, X. Chen, and K. Huang, "Design of microstrip reflectarray antenna using a genetic algorithm based optimization method," Electromagnetics, vol. 32, no. 2, pp. 77-85, 2012.

[18] D. M. Pozar and T. A. Metzler, "Analysis of a reflectarray antenna using microstrip patches of variable size," Electronics Letters, vol. 29, no. 8, pp. 657-658, 1993. 


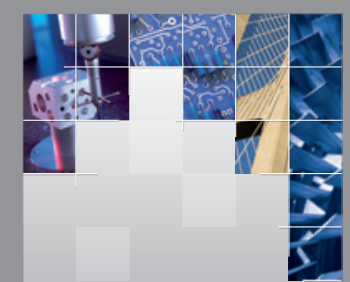

\section{Enfincering}
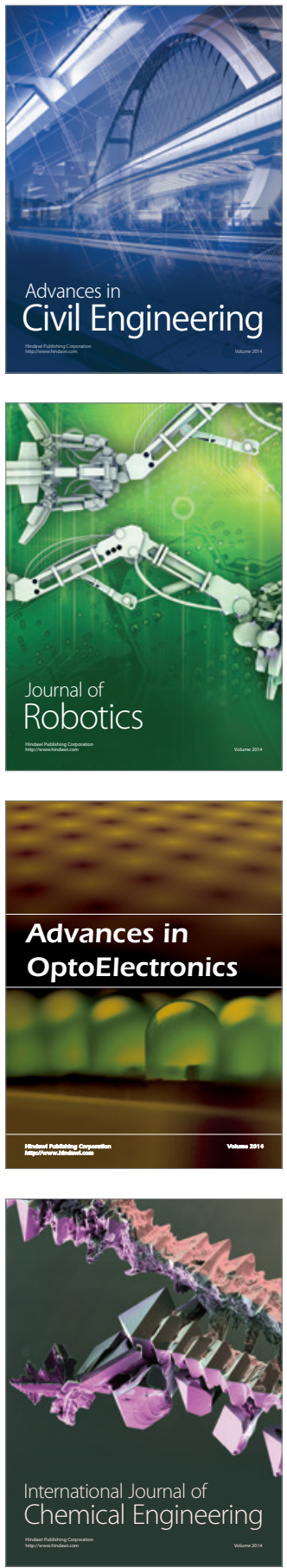

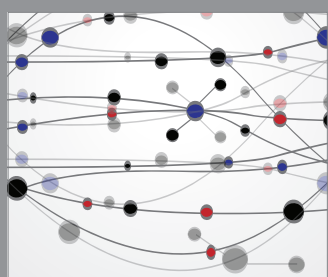

The Scientific World Journal

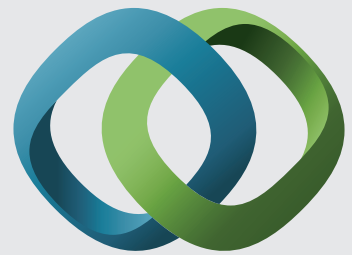

\section{Hindawi}

Submit your manuscripts at

http://www.hindawi.com
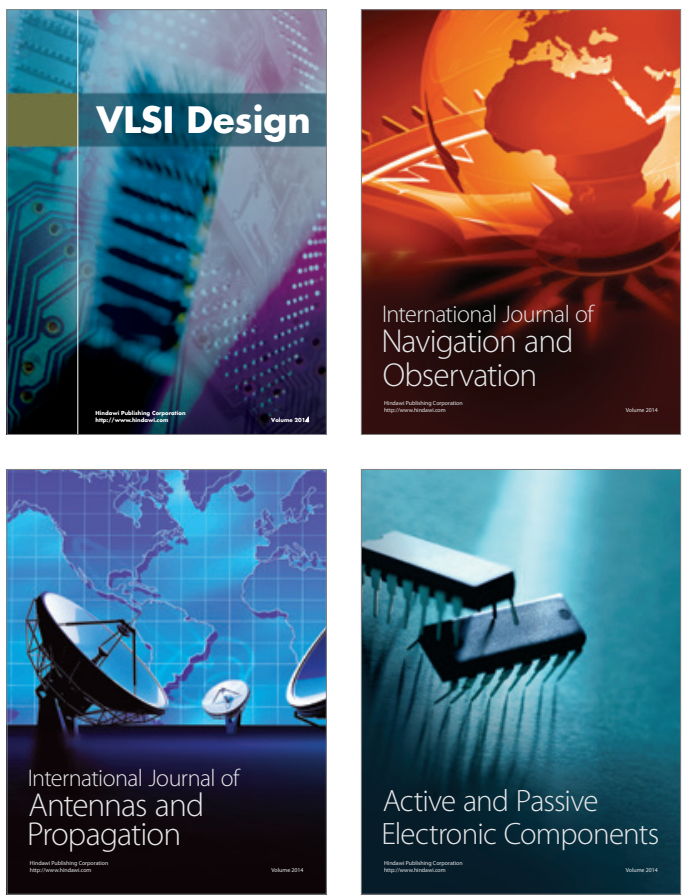
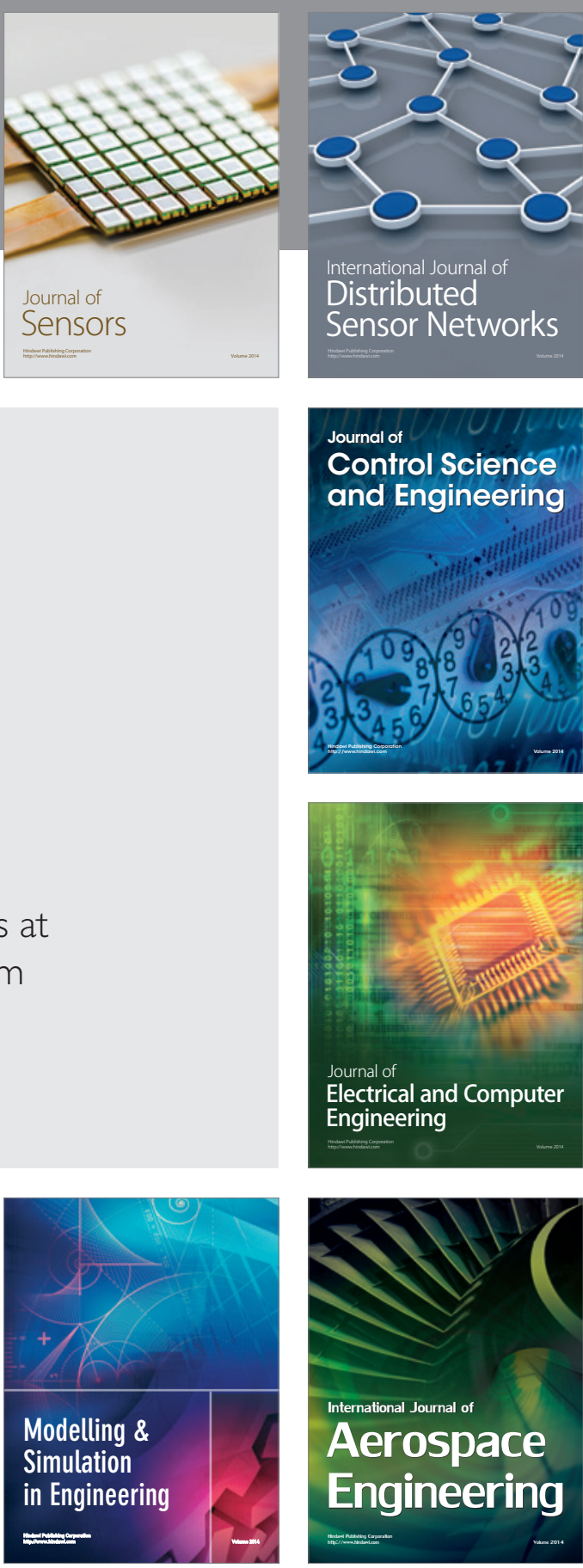

International Journal of

Distributed

Sensor Networks

Journal of

Control Science

and Engineering
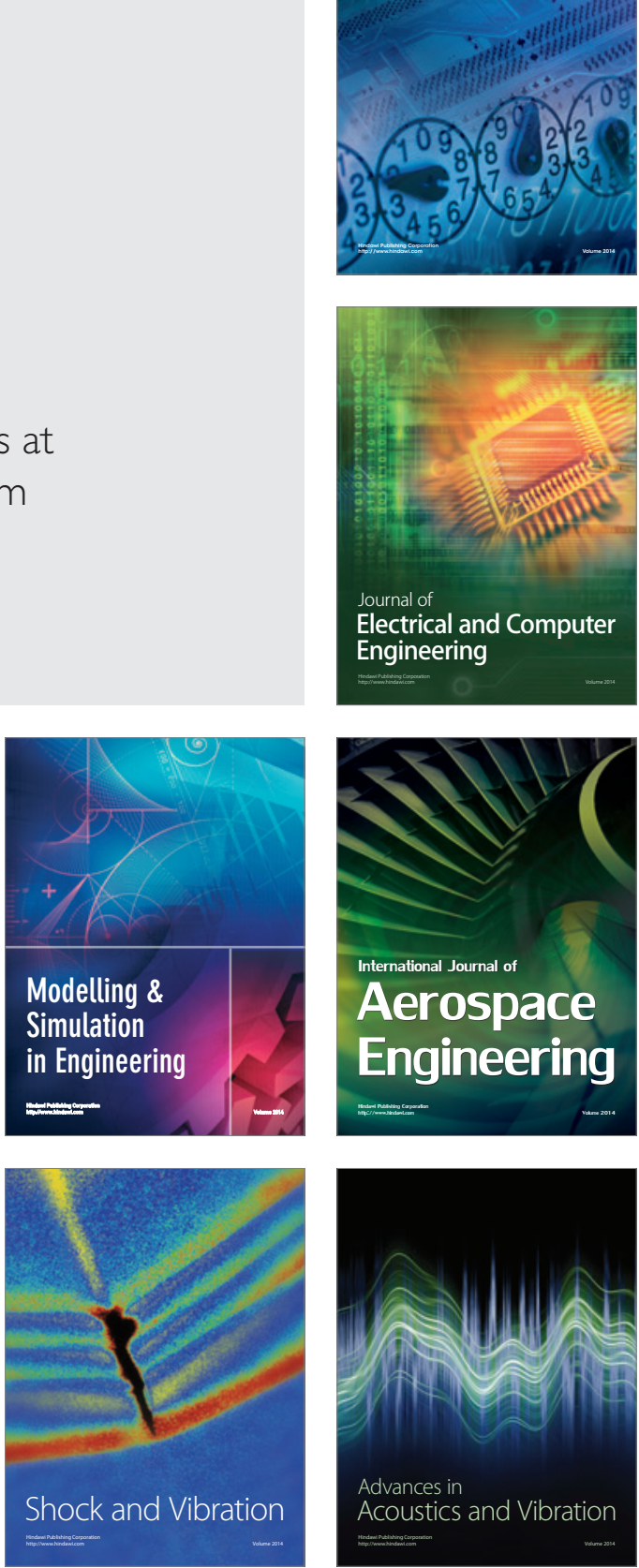